\title{
Experimental Verification of the Work Fluctuation-Dissipation Relation for Information-to-Work Conversion
}

\author{
David Barker $\odot,{ }^{1, *}$ Matteo Scandi $\odot,{ }^{2}$ Sebastian Lehmann, ${ }^{1}$ Claes Thelander $\odot,{ }^{1}$ \\ Kimberly A. Dick, ${ }^{1,3}$ Martí Perarnau-Llobet $\oplus^{4},{ }^{4}$ and Ville F. Maisi $\oplus^{1, \dagger}$ \\ ${ }^{1}$ NanoLund and Solid State Physics, Lund University, Box 118, 22100 Lund, Sweden \\ ${ }^{2}$ ICFO_Institut de Ciencies Fotoniques, The Barcelona Institute of Science and Technology, \\ Castelldefels (Barcelona) 08860, Spain \\ ${ }^{3}$ Centre for Analysis and Synthesis, Lund University, Box 124, 22100 Lund, Sweden \\ ${ }^{4}$ Département de Physique Appliquée, Université de Genéve, 1211 Genéve, Switzerland
}

(Received 7 September 2021; revised 23 November 2021; accepted 22 December 2021; published 27 January 2022)

\begin{abstract}
We study experimentally work fluctuations in a Szilard engine that extracts work from information encoded as the occupancy of an electron level in a semiconductor quantum dot. We show that as the average work extracted per bit of information increases toward the Landauer limit $k_{B} T \ln 2$, the work fluctuations decrease in accordance with the work fluctuation-dissipation relation. We compare the results to a protocol without measurement and feedback and show that when no information is used, the work output and fluctuations vanish simultaneously, contrasting the information-to-energy conversion case where increasing amount of work is produced with decreasing fluctuations. Our study highlights the importance of fluctuations in the design of information-to-work conversion processes.
\end{abstract}

DOI: 10.1103/PhysRevLett.128.040602

When considering thermodynamic processes at the microscale, where fluctuations play a dominant role, one faces the challenge of describing work and heat as stochastic quantities. Understanding and characterizing their statistics has become an active area of research and is one of the main aims of the field of stochastic thermodynamics [1,2]. The exact form of heat and work distributions highly depends on the specific protocol in question. The situation considerably simplifies when considering finite-time processes where the system remains close to the instantaneous equilibrium state (i.e., in the linear-response regime with respect to the driving speed) [3-11]. In this case, and when no quantum coherence is generated along the process, the work distribution takes a Gaussian shape $[4,6,10]$, with its first and second moments related through the work fluctuation-dissipation relation (FDR) [12-15]

$$
W_{\mathrm{diss}}=\frac{1}{2 k_{B} T} \sigma_{W}^{2}
$$

Here, $T$ is the temperature of the surrounding environment, $k_{B}$ is Boltzmann's constant, $W_{\text {diss }}$ is the average dissipated work along the process

Published by the American Physical Society under the terms of the Creative Commons Attribution 4.0 International license. Further distribution of this work must maintain attribution to the author(s) and the published article's title, journal citation, and DOI. Funded by Bibsam.

$$
W_{\text {diss }}:=\langle W\rangle-\Delta F,
$$

i.e., the difference between the mean work $\langle W\rangle$ performed to the system and the change in equilibrium free energy $\Delta F$, and finally $\sigma_{W}^{2}$ is the variance of the work distribution. The work FDR is a central result in stochastic thermodynamics: it implies that near equilibrium, dissipation comes inevitably accompanied by fluctuations, and conversely that optimal protocols minimizing dissipation will automatically also minimize fluctuations [5,16]. The FDR also plays a crucial role in the estimation of equilibrium free energy differences through measurements of nonequilibrium processes $[17,18]$.

The main goal of this Letter is to experimentally validate the fundamental relation, Eq. (1), by using an electronic system. For that, we consider one of the most relevant thermodynamic processes at the nanoscale: the conversion of information into work. Inspired by the Szilard engine, we implement an information engine that uses one bit of information (an extra electron being located on the quantum dot or not) to extract work $W_{\text {ex }} \equiv-W$ from a thermal reservoir at temperature $T$. This cycle and its inverse, the so-called Landauer erasure, have been extensively studied in the literature, both theoretically [19-30] and experimentally [31-36]. It is well known that the average extractable work is bounded by the so-called Landauer limit $\left\langle W_{\mathrm{ex}}\right\rangle \leq k_{B} T \ln 2$, which can only be reached for infinitesimally slow processes. Previous experimental important works have successfully managed to approach this limit to a high degree in various platforms at the level of average 
work [31-36]. In contrast, here we focus our attention on the behavior of work fluctuations, and in particular show how Eq. (1) is satisfied as the Landauer limit is approached. As predicted from the FDR, our results clearly demonstrate a decrease in work fluctuations as the dissipation is minimized by increasing the operation cycle time of the engine. Therefore, the information about the electron's position can be exploited to extract work in an almostdeterministic fashion. To contrast this information-to-work process, we consider a cyclic process where no information on the electron's position is available. In this case, we also observe that both work fluctuations and dissipation decrease according to Eq. (1) as the operating time of the cycle increases. However, the average extracted work always remains negative, $\left\langle W_{\mathrm{ex}}\right\rangle \leq 0$, and approaches zero for long cycle times, in agreement with the second law of thermodynamics. This contrasts with the information engine that provides a positive finite work output with fluctuations vanishing toward long cycle times.

Our device is based on a semiconductor quantum dot that allows for the manipulation of discrete energy levels as opposed to the metallic system used in Ref [35]. This work thus provides an experimental insight into the nature of thermodynamic fluctuations in nanoscale systems as well as an alternative platform to study information-to-work conversion with high efficiency.

Experimental setup.-Our device is shown in Fig. 1(a). It consists of a quantum dot (QD) system formed with polytypes in an InAs nanowire [37-40]. The bit of information we use in our experiment is encoded in the occupancy of an electronic state in the leftmost QD (QD1). The state is at discrete energy level $E$, which we drive using the plunger gate voltage $V_{g 1}$ with the lever arm $\alpha=1.7 \times 10^{4} k_{B} T / \mathrm{V}$. The rightmost $\mathrm{QD}$ is voltage biased with $V_{b}$ and used as an electrometer that detects the occupancy $n$ of the state $[41,42]$ through the current $I_{d}$ as shown in Fig. 1(b). Meanwhile, the middle QD is put in Coulomb blockade allowing tunneling from QD1 only to the left electronic reservoir. As in Refs. $[35,43,44]$, our experiment is performed in a regime where $n \in\{0,1\}$ (i.e., the QD1 has an extra electron or not). $n$ switches whenever an electron tunnels between QD1, and the leftmost fermionic reservoir at temperature $T=100 \mathrm{mK}$, given by the cryostat temperature. The tunneling occurs with the rates $\Gamma_{\text {in }}=\Gamma_{0}(1+b E) f(E)$ and $\Gamma_{\text {out }}=2 \Gamma_{0}[1+b E][1-f(E)]$ where $\Gamma_{0}=64 \mathrm{~Hz}$ and $b=-0.025 / k_{B} T$ were determined using a feedback protocol developed in Ref. [45], and $f(E)=1 /\left[1+e^{\beta E}\right]$ is the Fermi-Dirac distribution with $\beta=1 / k_{B} T$.

In the experiment, we consider protocols where the energy $E(t)$ is driven linearly from $E(0) \equiv E_{i}$ to $E(\tau) \equiv E_{f}$ for a time $\tau$. Work $W$ is performed on the system whenever $n(t)=1$ during the drive, i.e., $W \equiv \int_{0}^{\tau} d t n(t) \dot{E}(t)$. Note that work is a stochastic quantity due to fluctuations in $n(t)$ $[1,2,46-48]$. Since $n(t)$ is measured and $\dot{E}(t)$ is determined (a)

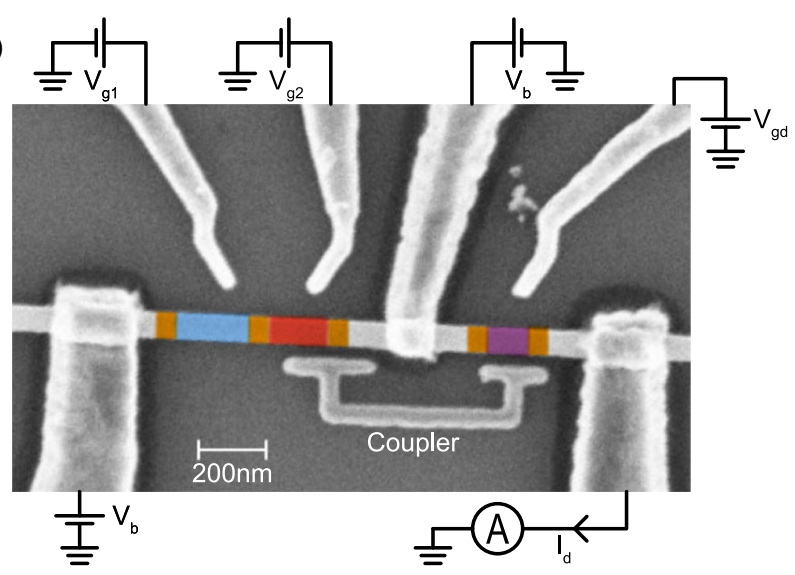

(b)

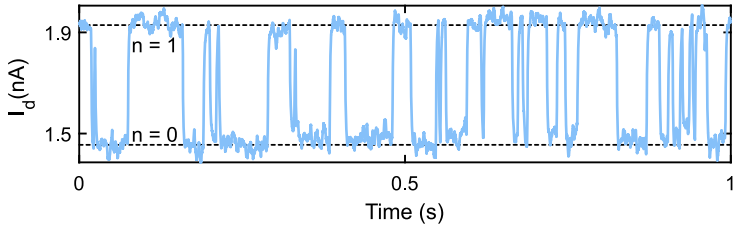

FIG. 1. (a) Scanning electron microscope image of the nanowire device. Embedded in the nanowire are three QDs, each aligned to one of the plunger gates $V_{g 1}, V_{g 2}$, or $V_{g d}$. Contacts separate the device into one part with two QDs and one part with a single QD. The coupler couples the two systems together, allowing the current $I_{d}$ through the lone QD to provide a measure of the charge state of the other system. Here, the QD involved in the experiment is marked in blue (close to the plunger gate with $V_{g 1}$ ) while the quantum dot marked in red is put in Coulomb blockade. The sensor QD is marked in purple and the tunnel barriers are colored orange. (b) An example of the sensor current $I_{d}$ at the start of the experiment. $V_{g d}$ is set so that a high current represents the state $n=1$ and $V_{g 1}$ is set so that the average $\langle n\rangle=0.5$.

by the drive we apply using $V_{g 1}$, the above equation gives us $W$ for a given realization of the experiment. Sufficient repetitions of the protocol enable us to obtain the work probability distribution $P(W)$ experimentally. With the distribution, we obtain directly the average $\langle W\rangle$ and the standard deviation $\sigma_{W}$ to test Eqs. (1) and (2). The free energy change for the information engine case is $\Delta F=$ $k_{B} T \ln 2$ since the protocol drives the system between equal probability for $n=0,1$, and a state far enough in energy $E$ so that only one state is allowed as described below. For the process not using information $\Delta F=0$, as it completes a cycle without using information. Further details about the experimental setup can be found in the Supplemental Material [49].

Information-to-work conversion.-The device is operated as a Szilard engine and extracts work $W_{\mathrm{ex}}=-W$ using the protocol developed in Ref. [35] and shown in Fig. 2(a). The protocol consists of three steps. (1) The QD is set to the condition of Fig. 1(b) where there is an equal chance to have $n=0$ and $n=1$, which occurs for a 

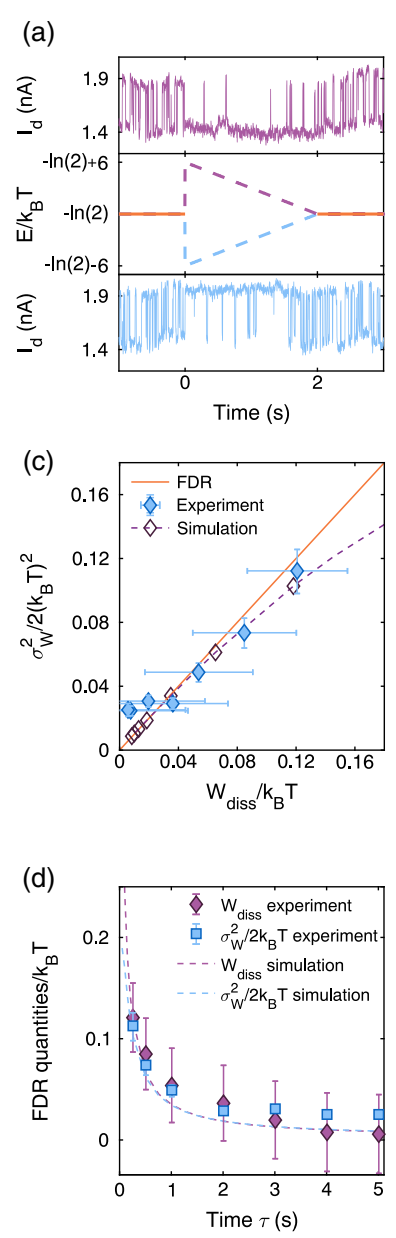

FIG. 2. (a) The middle panel illustrates how the energy level is driven during the information-to-work conversion. Originally, it is offset from the reservoir Fermi level by $E=-k_{B} T \ln 2$. At time 0 , a measurement is made. Depending on the outcome, the energy is rapidly driven either up or down by $6 k_{B} T$ before slowly being driven back to the starting point. In this example, the protocol time $\tau=2 \mathrm{~s}$. The upper (lower) panel shows the detector signal during one realization of the protocol where the energy was initially driven up (down). (b) The distributions of extracted work for the Szilard engine operation for the protocol lengths $\tau=0.25 \mathrm{~s}$ (top), $2 \mathrm{~s}$ (middle), and $5 \mathrm{~s}$ (bottom) together with the theoretically expected distribution (purple). The dotted black line indicates the Landauer limit $k_{B} T \ln 2$ and the orange dashed line indicates the mean extracted work. (c) The two quantities from Eq. (1) plotted against each other. Here, $W_{\text {diss }}=k_{B} T \ln 2-\left\langle W_{\text {ex }}\right\rangle$. The FDR line is Eq. (1), whereas the simulations are obtained by a full counting statistics method with Eq. (3). (d) Same quantities as in (c), but plotted against $\tau$. For details on the computation of error bars in (c) and (d), see the Supplemental Material [49].

doubly spin-degenerate energy level when $E=-k_{B} T \ln 2$. (2) A measurement of $n$ is made. This provides one bit of information, which is used to extract work. (3) Depending on the measurement outcome, one of two feedback cycles is applied using $V_{g 1}$ (the top and bottom panels of Fig. 2(a) show example realizations of the two choices): (a) If $n=0$,

the level is rapidly raised by amplitude $A=+6 k_{B} T$. This process does no work to the system as the energy level is empty. The energy level is then slowly ramped down to the starting point for a time $\tau$, extracting at most $\left\langle W_{\mathrm{ex}}\right\rangle=k_{B} T \ln 2$. (b) If $n=1$, the level is instead lowered by the drive amplitude, extracting $6 k_{B} T$ of work and locking the state in $n=1$. While ramping the energy back up, on average $\langle W\rangle \geq 6 k_{B} T-k_{B} T \ln 2$ of work is performed to the system.

In both cases, the total amount of average work extracted in the cycle is bounded by $\left\langle W_{\text {ex }}\right\rangle \leq k_{B} T \ln 2$.

We consider the performance of the information-to-work protocol for different times $\tau$, which we vary between $0.25 \mathrm{~s}$ and $5 \mathrm{~s}$. For each $\tau$, we perform the cycle on the order of $N \approx 1000$ times to obtain a distribution of extracted work $W_{\text {ex }}$. Figure 2(b) shows how the distribution of extracted work changes as $\tau$ is increased. In the short time limit, there is a large peak at $W_{\mathrm{ex}}=0$ that arises from instances where no tunneling events had time to occur. As the drive is made slower, these cases become less likely and the distribution starts forming a Gaussian distribution approaching the Landauer limit $k_{B} T \ln 2$. As we approach the limit, the distribution also sharpens (decreasing its variance $\sigma_{W}^{2}$ ), and the work FDR also becomes valid, as demonstrated in Figs. 2(c) and 2(d) [55]. Figure 2(c) shows the quantities of each side of Eq. (1) plotted against each other. The points line up with the diagonal, indicating that the work FDR does indeed hold. Furthermore, as seen in Fig. 2(d), both $\sigma_{W}^{2}$ and $W_{\text {diss }}$ agree with theoretical simulations (for details, see below and the Supplemental Material [49]). In general, we observe very good agreement between theory and simulations. However, with largest $\tau$ 's, $\sigma_{W}^{2}$ saturates in the experiment. This arises from offset charge drifts near QD1 that we observed to change at these timescales.

Dissipative drive.-We now contrast the information-towork conversion results with a protocol where no information is involved, and hence only dissipation is created. The protocol is presented in Fig. 3(a). Like the Szilard engine case, it also starts at $E=-k_{B} T \ln 2$ with a $50 \%$ chance of QD1 being occupied. However, instead of making a measurement and quickly driving the level, it is slowly ramped down by the drive amplitude $2.5 k_{B} T$ and then slowly ramped back up. The drive period $\tau$ is varied between $0.5 \mathrm{~s}$ and $4 \mathrm{~s}$, and Fig. 3(b) shows the work distribution in those limits as well as an intermediate case. In this case, $W_{\text {diss }}=\langle W\rangle$ where $W$ is the work done to the system during the drive. It is clear that as the drive slows down, $\langle W\rangle \rightarrow 0$, while the distribution also sharpens up, in accordance with the FDR. The validity of the FDR for this cycle is shown in Figs. 3(c) and 3(d), again finding very good agreement with experiment and theory expectations.

Figure 4 summarizes the results for all protocols tested. This includes the previously discussed information-toenergy conversion drive and the $2.5 k_{B} T$ dissipative drive as well as an additional dissipative drive with a $6 k_{B} T$ 

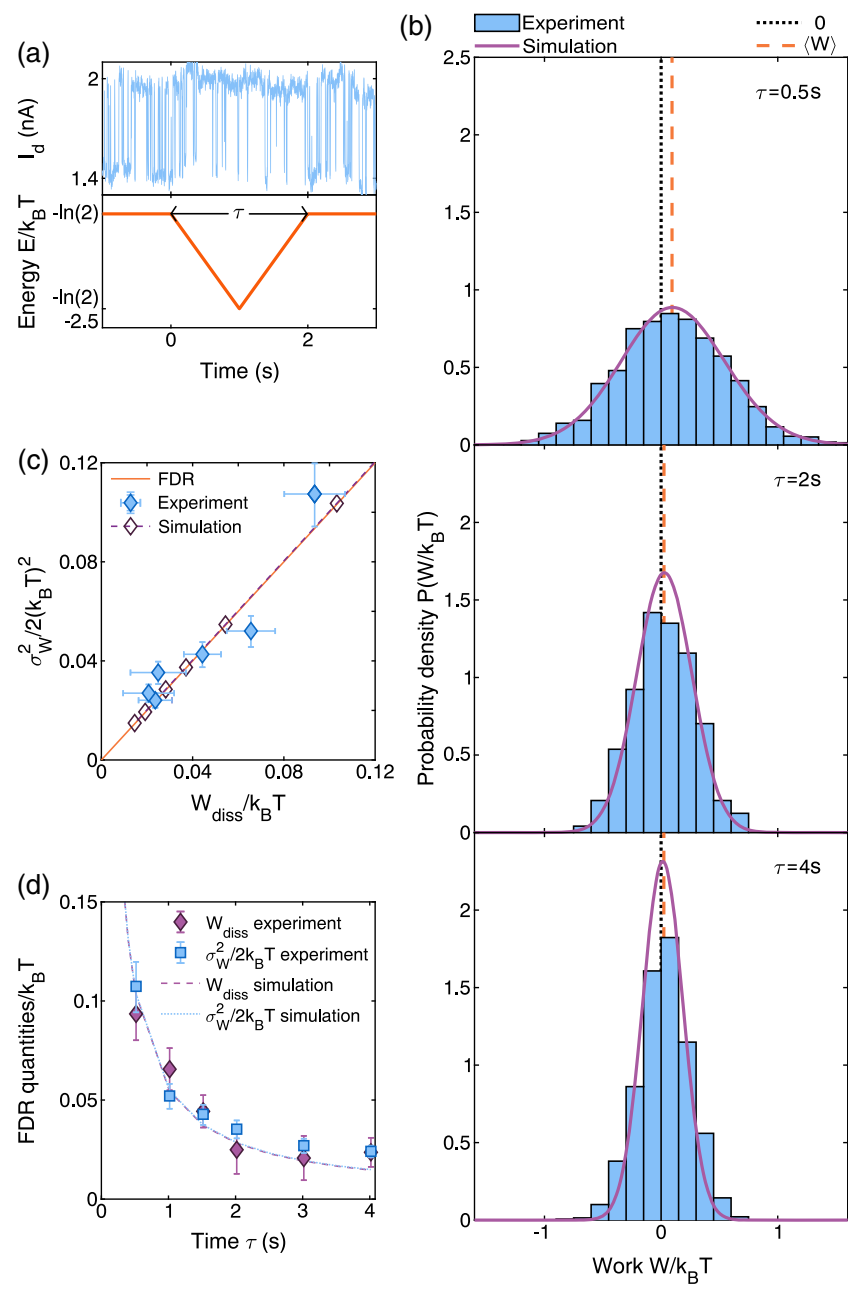

FIG. 3. (a) Top panel shows the detector signal $I_{d}$ during the dissipative drive protocol, which is shown in the bottom panel. In the dissipative drive, the energy level is initially offset from the reservoir Fermi energy by $E=-k_{B} T \ln 2$. Then, it is driven down by $2.5 k_{B} T$ and back up again during a time $\tau$. (b) The work distribution of the $2.5 k_{B} T$ dissipative drive for total protocol lengths $0.5 \mathrm{~s}$ (top), $2 \mathrm{~s}$, and $4 \mathrm{~s}$ (bottom) together with what is expected theoretically (purple). The orange line is the mean of the distribution, which in this case is equal to $W_{\text {diss }}$. The black line is drawn at $W=0$ to help guide the eye. (c) The two sides of Eq. (1) plotted against each other, for protocol lengths between $0.5 \mathrm{~s}$ and $4 \mathrm{~s}$. The orange diagonal is given by Eq. (4), which is obtained analytically in the slow-driving limit, and from which the FDR directly follows (see details in the Supplemental Material [49]). (d) The same quantities as in (c), but now plotted against the protocol length. For details on the computation of error bars in (c) and (d), see the Supplemental Material [49].

amplitude. All points follow Eq. (1), except the longest drives where the fluctuations saturate due to the offset charge drifts. These results encapsulate the main finding of this Letter: the experimental verification of the fundamental thermodynamic relation, Eq. (1), at the level of a single electron at a time occupying a discrete energy level in quantum dot.

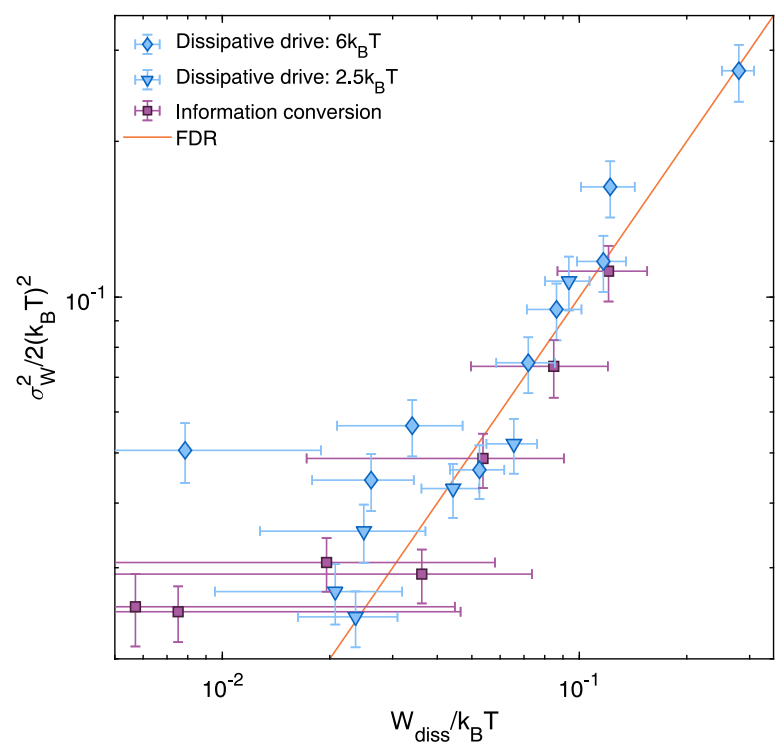

FIG. 4. The quantities in the work FDR of Eq. (1) plotted against each other for all protocols tested. This summarizes the data from the information conversion protocol (purple squares) and the dissipative drive with no information involved (blue triangles) plotted in Figs. 2(d) and 3(d), respectively. Blue diamonds represent a dissipative drive with an amplitude of $6 k_{B} T$ but otherwise identical to the $2.5 k_{B} T$ dissipative drive. For all protocols implemented, the points follow the orange line given by Eq. (1) up to the long time saturation of $\sigma_{W}$.

Theoretical considerations.-To describe the system theoretically, we made numerical simulations presented in Figs. 2 and 3 with the following formalism: During the drive, the average occupancy $p(t)$ of the QD state is governed by the rate equation

$$
\dot{p}(t)=\Gamma_{\text {in }}[1-p(t)]-\Gamma_{\text {out }} p(t) .
$$

This rate equation enables us to theoretically find $P(W)$ through the computation of the characteristic function, as detailed in the Supplemental Material [49]. Furthermore, in the Supplemental Material we also derive the following analytic expression for the dissipated work $W_{\text {diss }}$ and work fluctuations $\sigma_{W}^{2}$ :

$$
\begin{aligned}
W_{\mathrm{diss}} & =\beta \int_{0}^{\tau} \mathrm{d} t\left[1-p^{\mathrm{eq}}(t)\right] p^{\mathrm{eq}}(t) \frac{\dot{E}(t)^{2}}{\Gamma(t)}+\mathcal{O}\left(\frac{1}{\tau \Gamma_{0}}\right)^{2} \\
\sigma_{W}^{2} & =2 \frac{W_{\text {diss }}}{\beta}+\mathcal{O}\left(\frac{1}{\tau \Gamma_{0}}\right)^{2}
\end{aligned}
$$

where we have introduced $p^{\mathrm{eq}}(t)=1 /\left[1+2 e^{\beta E(t)}\right]$ and $\Gamma(t)=\Gamma_{0}[1+b E(t)]$. In Eq. (4) we have neglected contributions of order $\left(\tau \Gamma_{0}\right)^{-2}$, which is well justified in the experiment for $\tau \gtrsim 1 \mathrm{~s}$ (for $\tau=1 \mathrm{~s}$ we have $1 / \tau \Gamma_{0} \approx 0.16$ ). Equation (4) shows that the FDR is satisfied at order $1 / \tau \Gamma_{0}$, which is in good agreement with the experimental results, as well as the more general theoretical considerations of 
Refs. [14] and [15]. The regime where $W_{\text {diss }}$ (and $\sigma_{W}^{2}$ ) decay with $1 / \tau$ is often known in the literature as the lowdissipation regime; see, e.g., Refs. [56-61].

Conclusions. - In summary, we experimentally verified the work FDR for an information-to-work engine implemented in a semiconductor quantum dot system. In agreement with the FDR, we observed a sharpening of the work distribution as the Landauer or reversible limit is approached. This implies that $k_{B} T \ln 2$ can be extracted almost deterministically from one bit of information (the electron's position in the dot). We contrasted these results with a process where no information about the electron was used. In this case, we also observed a decrease of dissipation accompanied by a decrease in fluctuations, but no work could be extracted on average.

Overall, our results highlight that the fundamental thermodynamic relation, Eq. (1), is valid at the level of a single electron. These measurements constitute the first steps of studying information-to-work conversion using semiconductor quantum dots. Similar devices could be used to study, for instance, optimal protocols for information-to-work conversion [30,62-66], as well as thermodynamic uncertainty relations [67]. Another future direction of interest is to, instead of inferring work output from the QD occupation, use information to generate a current and measure the work output directly [68].

Finally, a longer-term challenge is to experimentally show violations of the work FDR of Eq. (1) due to the presence of quantum coherence, as theoretically predicted in Ref. [15], thus showing a genuine quantum signature in thermodynamics [10,46-48]. This could be achieved by studying a charge qubit consisting of two coupled states of the two QDs. However, since the coherence time in such qubits is at most on the order of $100 \mathrm{~ns}$ [69-71], a much faster readout would be required compared to the millisecond timescale in the present study.

We would like to thank Christopher Jarzynski for helpful discussions. D. B. and V.F.M. acknowledge financial support from NanoLund, Swedish Research Council (Dnr 2019-04111), grant number FQXi-IAF19-07 from the Foundational Questions Institute Fund, a donor advised fund of Silicon Valley Community Foundation and the Knut and Alice Wallenberg Foundation (KAW) via Project No. 2016.0089. M. P.-L. acknowledges funding from Swiss National Science Foundation through an Ambizione Grant No. PZ00P2-186067. M. S. acknowledges support from the European Union's Horizon 2020 research and innovation programme under the Marie Skłodowska-Curie Grant Agreement No. 713729, and from the Government of Spain (FIS2020-TRANQI and Severo Ochoa CEX2019-000910- S), Fundacio Cellex, Fundació Mir-Puig, Generalitat de Catalunya (SGR 1381 and CERCA Programme).
*Corresponding author. david.barker@ftf.lth.se Corresponding author. ville.maisi@ftf.lth.se

[1] C. Jarzynski, Equalities and inequalities: Irreversibility and the second law of thermodynamics at the nanoscale, Annu. Rev. Condens. Matter Phys. 2, 329 (2011).

[2] U. Seifert, Stochastic thermodynamics, fluctuation theorems and molecular machines, Rep. Prog. Phys. 75, 126001 (2012).

[3] J. Nulton, P. Salamon, B. Andresen, and Q. Anmin, Quasistatic processes as step equilibrations, J. Chem. Phys. 83, 334 (1985).

[4] T. Speck and U. Seifert, Distribution of work in isothermal nonequilibrium processes, Phys. Rev. E 70, 066112 (2004).

[5] G. E. Crooks, Measuring Thermodynamic Length, Phys. Rev. Lett. 99, 100602 (2007).

[6] J. Hoppenau and A. Engel, On the work distribution in quasi-static processes, J. Stat. Mech. (2013) P06004.

[7] C. Kwon, J. D. Noh, and H. Park, Work fluctuations in a time-dependent harmonic potential: Rigorous results beyond the overdamped limit, Phys. Rev. E 88, 062102 (2013).

[8] M. V.S. Bonança and S. Deffner, Optimal driving of isothermal processes close to equilibrium, J. Chem. Phys. 140, 244119 (2014).

[9] D. Mandal and C. Jarzynski, Analysis of slow transitions between nonequilibrium steady states, J. Stat. Mech. (2016) 063204.

[10] M. Scandi, H. J. D. Miller, J. Anders, and M. PerarnauLlobet, Quantum work statistics close to equilibrium, Phys. Rev. Research 2, 023377 (2020).

[11] B. Bhandari, P. T. Alonso, F. Taddei, F. von Oppen, R. Fazio, and L. Arrachea, Geometric properties of adiabatic quantum thermal machines, Phys. Rev. B 102, 155407 (2020).

[12] J. Hermans, Simple analysis of noise and hysteresis in (slow-growth) free energy simulations, J. Phys. Chem. 95, 9029 (1991).

[13] C. Jarzynski, Nonequilibrium Equality for Free Energy Differences, Phys. Rev. Lett. 78, 2690 (1997).

[14] D. Mandal and C. Jarzynski, Analysis of slow transitions between nonequilibrium steady states, J. Stat. Mech. (2016) 063204.

[15] H. J. D. Miller, M. Scandi, J. Anders, and M. PerarnauLlobet, Work Fluctuations in Slow Processes: Quantum Signatures and Optimal Control, Phys. Rev. Lett. 123, 230603 (2019).

[16] D. A. Sivak and G. E. Crooks, Thermodynamic Metrics and Optimal Paths, Phys. Rev. Lett. 108, 190602 (2012).

[17] G. Hummer, Fast-growth thermodynamic integration: Error and efficiency analysis, J. Chem. Phys. 114, 7330 (2001).

[18] C. Dellago and G. Hummer, Computing equilibrium free energies using non-equilibrium molecular dynamics, Entropy 16, 41 (2013).

[19] R. Landauer, Irreversibility and heat generation in the computing process, IBM J. Res. Dev. 5, 183 (1961).

[20] R. Landauer, Dissipation and noise immunity in computation and communication, Nature (London) 335, 779 (1988). 
[21] T. Sagawa and M. Ueda, Second Law of Thermodynamics with Discrete Quantum Feedback Control, Phys. Rev. Lett. 100, 080403 (2008).

[22] T. Sagawa and M. Ueda, Generalized Jarzynski Equality Under Nonequilibrium Feedback Control, Phys. Rev. Lett. 104, 090602 (2010).

[23] J. M. Horowitz and J. M. R. Parrondo, Thermodynamic reversibility in feedback processes, Europhys. Lett. 95, 10005 (2011).

[24] D. Mandal and C. Jarzynski, Work and information processing in a solvable model of Maxwell's demon, Proc. Natl. Acad. Sci. U.S.A. 109, 11641 (2012).

[25] A. C. Barato and U. Seifert, An autonomous and reversible maxwell's demon, Europhys. Lett. 101, 60001 (2013).

[26] S. Deffner and C. Jarzynski, Information Processing and the Second Law of Thermodynamics: An Inclusive, Hamiltonian Approach, Phys. Rev. X 3, 041003 (2013).

[27] J. M. R. Parrondo, J. M. Horowitz, and T. Sagawa, Thermodynamics of information, Nat. Phys. 11, 131 (2015).

[28] P. Faist and R. Renner, Fundamental Work Cost of Quantum Processes, Phys. Rev. X 8, 021011 (2018).

[29] H. J. D. Miller, G. Guarnieri, M. T. Mitchison, and J. Goold, Quantum Fluctuations Hinder Finite-Time Information Erasure Near the Landauer Limit, Phys. Rev. Lett. 125, 160602 (2020).

[30] K. Proesmans, J. Ehrich, and J. Bechhoefer, Finite-Time Landauer Principle, Phys. Rev. Lett. 125, 100602 (2020).

[31] A. Brut, A. Arakelyan, A. Petrosyan, S. Ciliberto, R. Dillenschneider, and E. Lutz, Experimental verification of Landauer's principle linking information and thermodynamics, Nature (London) 483, 187 (2012).

[32] V. Serreli, C. F. Lee, E. Kay, and D. A. Leigh, A molecular information ratchet, Nature (London) 445, 523 (2007).

[33] M. G. Raizen, Comprehensive control of atomic motion, Science 324, 1403 (2009).

[34] S. Toyabe, T. Sagawa, M. Ueda, E. Muneyuki, and M. Sano, Experimental demonstration of information-to-energy conversion and validation of the generalized Jarzynski equality, Nat. Phys. 6, 988 (2010).

[35] J. V. Koski, V. F. Maisi, J. P. Pekola, and D. V. Averin, Experimental realization of a Szilard engine with a single electron, Proc. Natl. Acad. Sci. U.S.A. 111, 13786 (2014).

[36] J. V. Koski, A. Kutvonen, I. M. Khaymovich, T. Ala-Nissila, and J. P. Pekola, On-Chip Maxwell's Demon as an Information-Powered Refrigerator, Phys. Rev. Lett. 115, 260602 (2015).

[37] S. Lehmann, J. Wallentin, D. Jacobsson, K. Deppert, and K. A. Dick, A general approach for sharp crystal phase switching in InAs, GaAs, InP and GaP nanowires using only group V flow, Nano Lett. 13, 4099 (2013).

[38] M. Nilsson, L. Namazi, S. Lehmann, M. Leijnse, K. A. Dick, and C. Thelander, Single-electron transport in InAs nanowire quantum dots formed by crystal phase engineering, Phys. Rev. B 93, 195422 (2016).

[39] I.-J. Chen, S. Lehmann, M. Nilsson, P. Kivisaari, H. Linke, K. A. Dick, and C. Thelander, Conduction band offset and polarization effects in InAs nanowire polytype junctions, Nano Lett. 17, 902 (2017).

[40] D. Barker, S. Lehmann, L. Namazi, M. Nilsson, C. Thelander, K. A. Dick, and V.F. Maisi, Individually addressable double quantum dots formed with nanowire polytypes and identified by epitaxial markers, Appl. Phys. Lett. 114, 183502 (2019).

[41] L. M. K. Vandersypen, J. M. Elzerman, R. N. Schouten, L. H. W. van Beveren, R. Hanson, and L. P. Kouwenhoven, Real-time detection of single-electron tunneling using a quantum point contact, Appl. Phys. Lett. 85, 4394 (2004).

[42] S. Gustavsson, R. Leturcq, M. Studer, I. Shorubalko, T. Ihn, K. Ensslin, D. C. Driscoll, and A.C. Gossard, Electron counting in quantum dots, Surf. Sci. Rep. 64, 191 (2009).

[43] A. Hofmann, V. F. Maisi, J. Basset, C. Reichl, W. Wegscheider, T. Ihn, K. Ensslin, and C. Jarzynski, Heat dissipation and fluctuations in a driven quantum dot, Phys. Status Solidi (b) 254, 1600546 (2017).

[44] A. Hofmann, V.F. Maisi, C. Rössler, J. Basset, T. Krähenmann, P. Märki, T. Ihn, K. Ensslin, C. Reichl, and W. Wegscheider, Equilibrium free energy measurement of a confined electron driven out of equilibrium, Phys. Rev. B 93, 035425 (2016).

[45] A. Hofmann, V. F. Maisi, C. Gold, T. Krähenmann, C. Rössler, J. Basset, P. Märki, C. Reichl, W. Wegscheider, K. Ensslin, and T. Ihn, Measuring the Degeneracy of Discrete Energy Levels Using a GaAs/AlGaAs Quantum Dot, Phys. Rev. Lett. 117, 206803 (2016).

[46] M. Esposito, U. Harbola, and S. Mukamel, Nonequilibrium fluctuations, fluctuation theorems, and counting statistics in quantum systems, Rev. Mod. Phys. 81, 1665 (2009).

[47] M. Campisi, P. Hänggi, and P. Talkner, Erratum: Quantum fluctuation relations: Foundations and applications (Reviews of Modern Physics), Rev. Mod. Phys. 83, 771 (2011).

[48] J. Goold, M. Huber, A. Riera, L. del Rio, and P. Skrzypczyk, The role of quantum information in thermodynamics-a topical review, J. Phys. A 49, 143001 (2016).

[49] See Supplemental Material, which includes Refs. [50-54], at http://link.aps.org/supplemental/10.1103/PhysRevLett .128.040602 for details about device fabrication, measurements, data analysis, numerical methods, and a derivation of the FDR for the system in question.

[50] L. Namazi, M. Nilsson, S. Lehmann, C. Thelander, and K. A. Dick, Selective GaSb radial growth on crystal phase engineered InAs nanowires, Nanoscale 7, 10472 (2015).

[51] V. Cavina, A. Mari, and V. Giovannetti, Slow Dynamics and Thermodynamics of Open Quantum Systems, Phys. Rev. Lett. 119, 050601 (2017).

[52] M. Campisi, S. Denisov, and P. Hänggi, Geometric magnetism in open quantum systems, Phys. Rev. A 86, 032114 (2012).

[53] S. Deffner and E. Lutz, Thermodynamic length for far-fromequilibrium quantum systems, Phys. Rev. E 87, 022143 (2013).

[54] M. F. Ludovico, F. Battista, F. von Oppen, and L. Arrachea, Adiabatic response and quantum thermoelectrics for acdriven quantum systems, Phys. Rev. B 93, 075136 (2016).

[55] An important remark is that in order to study the work FDR, we focus only on instances where the correct feedback drive was applied. There is a possibility that an electron tunnel between the measurement is made and the feedback is applied, which gives rise to a second distribution centered 
around $k_{B} T(\ln 2-6)$. In this particular experiment, the error rate was roughly $3 \%$, but we expect to see fewer errors if the device is run in a regime with slower tunneling rates.

[56] B. Andresen, P. Salamon, and R. S. Berry, Thermodynamics in finite time, Phys. Today 37, No. 9, 62 (1984).

[57] M. Esposito, R. Kawai, K. Lindenberg, and C. Van den Broeck, Efficiency at Maximum Power of Low-Dissipation Carnot Engines, Phys. Rev. Lett. 105, 150603 (2010).

[58] A. C. Hernández, A. Medina, and J. M. M. Roco, Time, entropy generation, and optimization in low-dissipation heat devices, New J. Phys. 17, 075011 (2015).

[59] V. Holubec and A. Ryabov, Maximum efficiency of lowdissipation heat engines at arbitrary power, J. Stat. Mech. (2016) 073204.

[60] Y.-H. Ma, D. Xu, H. Dong, and C.-P. Sun, Universal constraint for efficiency and power of a low-dissipation heat engine, Phys. Rev. E 98, 042112 (2018).

[61] Y.-H. Ma, R.-X. Zhai, J. Chen, C. P. Sun, and H. Dong, Experimental Test of the $1 / \tau$-Scaling Entropy Generation in Finite-Time Thermodynamics, Phys. Rev. Lett. 125, 210601 (2020).

[62] M. Scandi and M. Perarnau-Llobet, Thermodynamic length in open quantum systems, Quantum 3, 197 (2019).

[63] P. Abiuso, H. J. D. Miller, M. Perarnau-Llobet, and M. Scandi, Geometric optimisation of quantum thermodynamic processes, Entropy 22, 1076 (2020).
[64] H. J. D. Miller and M. Mehboudi, Geometry of Work Fluctuations Versus Efficiency in Microscopic Thermal Machines, Phys. Rev. Lett. 125, 260602 (2020).

[65] Y.-Z. Zhen, D. Egloff, K. Modi, and O. Dahlsten, Universal Bound on Energy Cost of Bit Reset in Finite Time, Phys. Rev. Lett. 127, 190602 (2021).

[66] M. Konopik, T. Korten, E. Lutz, and H. Linke, Fundamental energy cost of finite-time computing, arXiv:2101.07075.

[67] P. P. Potts and P. Samuelsson, Thermodynamic uncertainty relations including measurement and feedback, Phys. Rev. E 100, 052137 (2019).

[68] B. Annby-Andersson, P. Samuelsson, V. F. Maisi, and P. P. Potts, Maxwell's demon in a double quantum dot with continuous charge detection, Phys. Rev. B 101, 165404 (2020).

[69] T. Hayashi, T. Fujisawa, H. D. Cheong, Y. H. Jeong, and Y. Hirayama, Coherent Manipulation of Electronic States in a Double Quantum Dot, Phys. Rev. Lett. 91, 226804 (2003).

[70] J. Gorman, D. G. Hasko, and D. A. Williams, Charge-Qubit Operation of an Isolated Double Quantum Dot, Phys. Rev. Lett. 95, 090502 (2005).

[71] P. Scarlino, D. J. van Woerkom, A. Stockklauser, J. V. Koski, M. C. Collodo, S. Gasparinetti, C. Reichl, W. Wegscheider, T. Ihn, K. Ensslin, and A. Wallraff, AllMicrowave Control and Dispersive Readout of GateDefined Quantum Dot Qubits in Circuit Quantum Electrodynamics, Phys. Rev. Lett. 122, 206802 (2019). 